This item was submitted to Loughborough's Research Repository by the author.

Items in Figshare are protected by copyright, with all rights reserved, unless otherwise indicated.

\title{
Semiconductor charge transport driven by a picosecond strain pulse
}

PLEASE CITE THE PUBLISHED VERSION

http://dx.doi.org/10.1063/1.2942389

\section{PUBLISHER}

(C) American Institute of Physics (AIP)

\section{VERSION}

VoR (Version of Record)

\section{LICENCE}

CC BY-NC-ND 4.0

\section{REPOSITORY RECORD}

Fowler, D.R., A.V. Akimov, Alexander G. Balanov, M.T. Greenaway, M. Henini, T.M. Fromhold, and A.J. Kent. 2019. "Semiconductor Charge Transport Driven by a Picosecond Strain Pulse". figshare.

https://hdl.handle.net/2134/12741. 
This item was submitted to Loughborough's Institutional Repository (https://dspace.lboro.ac.uk/) by the author and is made available under the following Creative Commons Licence conditions.

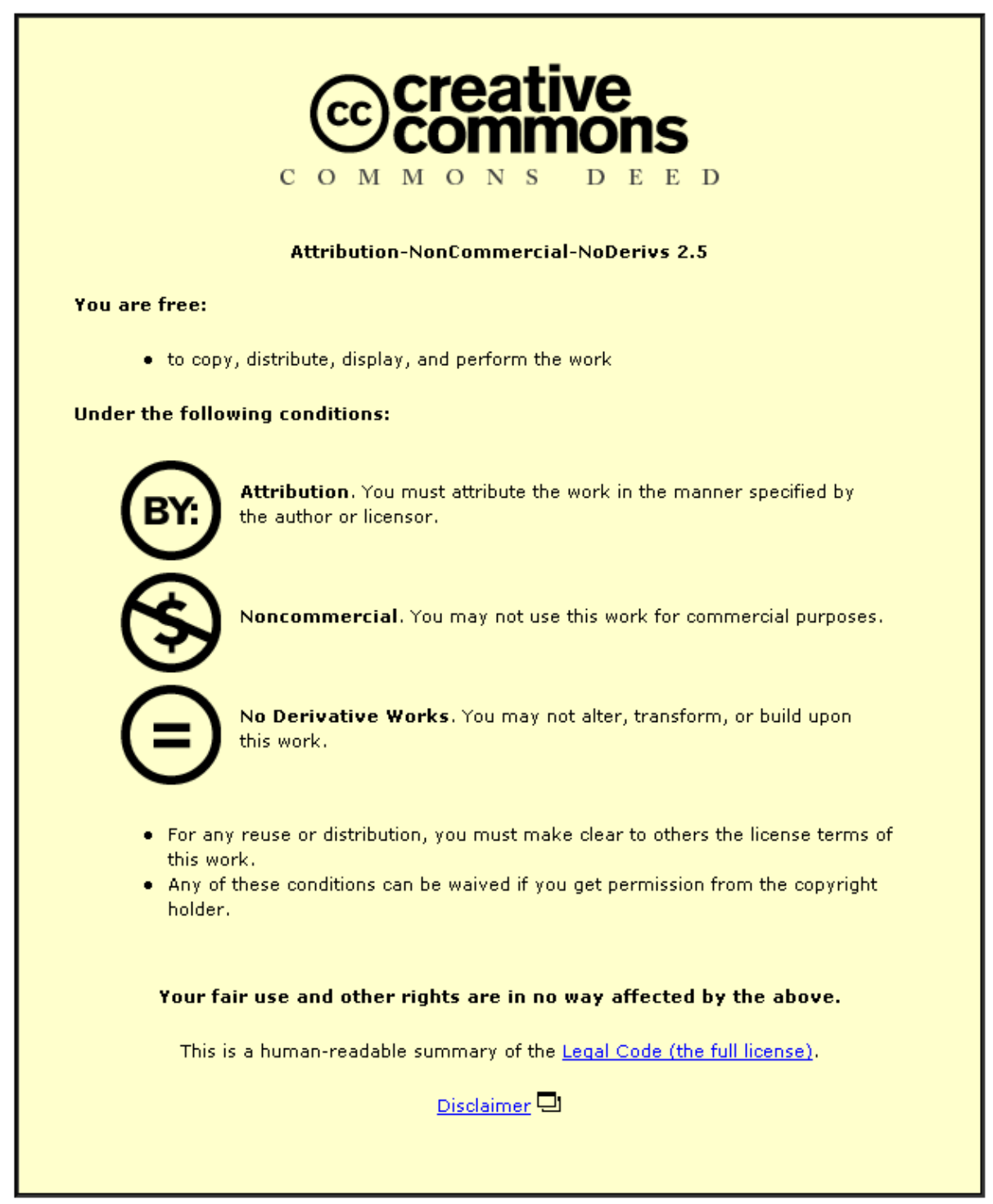

For the full text of this licence, please go to: http://creativecommons.org/licenses/by-nc-nd/2.5/ 


\title{
Semiconductor charge transport driven by a picosecond strain pulse
}

\author{
D. R. Fowler, ${ }^{1}{ }^{1, a)}$ A. V. Akimov, ${ }^{1}$ A. G. Balanov, ${ }^{2}$ M. T. Greenaway, ${ }^{1}$ M. Henini, ${ }^{1}$ \\ T. M. Fromhold, ${ }^{1}$ and A. J. Kent ${ }^{1}$ \\ ${ }^{1}$ School of Physics and Astronomy, University of Nottingham, Nottinghamshire NG7 2RD, United Kingdom \\ ${ }^{2}$ Department of Physics, Loughborough University, Loughborough LE11 3TU, United Kingdom
}

(Received 10 April 2008; accepted 19 May 2008; published online 11 June 2008)

\begin{abstract}
We demonstrate that a picosecond strain pulse can be used to drive an electric current through both thin-film epilayer and heterostructure semiconductor crystals in the absence of an external electric field. By measuring the transient current pulses, we are able to clearly distinguish the effects of the coherent and incoherent components of the acoustic packet. The properties of the strain induced signal suggest a technique for exciting picosecond current pulses, which may be used to probe semiconductor devices. (c) 2008 American Institute of Physics. [DOI: 10.1063/1.2942389]
\end{abstract}

The availability of reliable, high power femtosecond pulsed lasers has resulted in rapid development of coherent picosecond acoustics techniques for probing the acoustic properties of solids. Fast thermoelastic deformation of a thin metal film on the surface of a crystal, induced by subpicosecond optical excitation, produces a coherent acoustic strain pulse which travels through the crystal at the speed of longitudinal sound, $c_{\mathrm{LA}}$. The dynamics of such a strain pulse have been studied using various time-resolved "pump-probe" measurements, see, e.g., Refs. $1-5$, showing that in contrast to a heat pulse, ${ }^{6}$ this type of acoustic pulse is composed of coherent phonons whose spectral content typically reaches around $100-200 \mathrm{GHz}$.

Coherent strain pulses have been used to probe the acoustic properties of various solids, see, e.g., Refs. 7 and 8 as well as the optical properties of quantum well devices. ${ }^{9}$ However, this technique also has potential as a tool for studying electronic transport processes on ultrashort time scales.

In this paper, we show that a strain pulse containing coherent acoustic phonons can be used to drive an electric current through both $n$-type epilayer and superlattice semiconductor crystals without an applied electric field. This mechanism may provide a convenient method for the electronic detection of strain pulses, as well as sources of subpicosecond current pulses, which could be used to characterize the next generation of ultrafast transistors. Furthermore, the contrasting nature of the excitation and scattering dynamics of electrons that are acoustically, rather than electrically driven could lead to interesting transport effects in semiconductor nanostructures.

Figure 1 shows the experimental scheme. Vertical transport structures grown by molecular beam epitaxy on semiinsulating (100) GaAs substrates were studied. One, a $n$-type GaAs epilayer, consisted of a $300 \mathrm{~nm}$ Si-doped $\left(2 \times 10^{18} \mathrm{~cm}^{-3}\right)$ bottom GaAs contact layer, separated from an identical top contact by a $177.5 \mathrm{~nm}$ lightly Si-doped $\left(2 \times 10^{15} \mathrm{~cm}^{-3}\right) \mathrm{GaAs}$ layer. The second structure was the same, except the contacts were separated by a 177.5-nm-thick superlattice (SL) composed of 14 periods each of $10 / 2.5 \mathrm{~nm}$ of $\mathrm{GaAs} / \mathrm{Al}_{0.3} \mathrm{Ga}_{0.7} \mathrm{As}$ Si-doped uniformly at a density of $2 \times 10^{15} \mathrm{~cm}^{-3}$. Columnar device mesas

a) Author to whom correspondence should be addressed. Electronic mail: daivid.fowler@nottingham.ac.uk.
$200 \mu \mathrm{m}$ in diameter were produced that were electrically contacted using annealed InGe:Au Ohmic contacts. The reverse side of the substrate was polished flat before thermally evaporating a $100 \mathrm{~nm} \mathrm{Al} \mathrm{film.} \mathrm{All} \mathrm{measurements} \mathrm{were} \mathrm{made}$ at low temperature $(T=2 \mathrm{~K})$ in an optical-access cryostat. The Al film was excited using either $40 \mathrm{fs} 800 \mathrm{~nm}$ pulses from an amplified Ti:sapphire laser or $10 \mathrm{~ns} 532 \mathrm{~nm}$ pulses from a frequency-doubled $Q$-switched Nd:YLF (yttrium lithium fluoride) laser, both with a repetition rate of $5 \mathrm{KHz}$. The light was focused to a $200 \mu \mathrm{m}$ spot and directed opposite the device mesa using automated $x-y$ scanning mirrors. Neutral density filters were used to vary the incident pulse energy in a range up to $16 \mathrm{~mJ} / \mathrm{cm}^{2}$, above which the Al film was damaged.

Current pulses induced in the device were detected by amplifying and measuring the transient changes in voltage directly across the top and grounded bottom contacts to the active region. The output of the preamplifier led directly into a digitizer with a $500 \mathrm{MHz}$ analog bandwidth.

First, we consider the results obtained using the $n$-type GaAs epilayer sample. A typical current-time, $I(\tau)$, signal taken using $40 \mathrm{fs} 10 \mathrm{~mJ} / \mathrm{cm}^{-2}$ excitation is shown in the inset of Fig 1. The small negative peak at $\tau=0$ is due to weak optical excitation arising from scattered light falling onto the device mesa. After a delay of approximately $45 \mathrm{~ns}$, a voltage pulse is observed, indicating the presence of a transient electric current. The time delay is consistent with the time of flight of longitudinal acoustic (LA) phonons across the

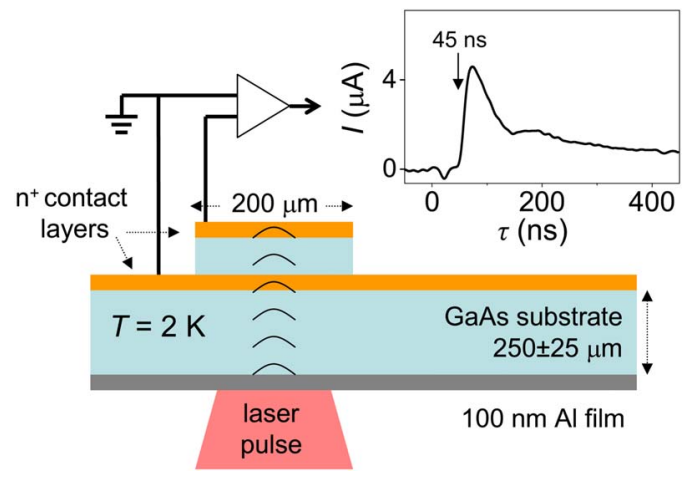

FIG. 1. (Color online) Experimental arrangement. Inset: typical $I(\tau)$ signal from a GaAs epilayer vertical device using a laser intensity of $10 \mathrm{~mJ} / \mathrm{cm}^{2}$ at $T=2 \mathrm{~K}$. 

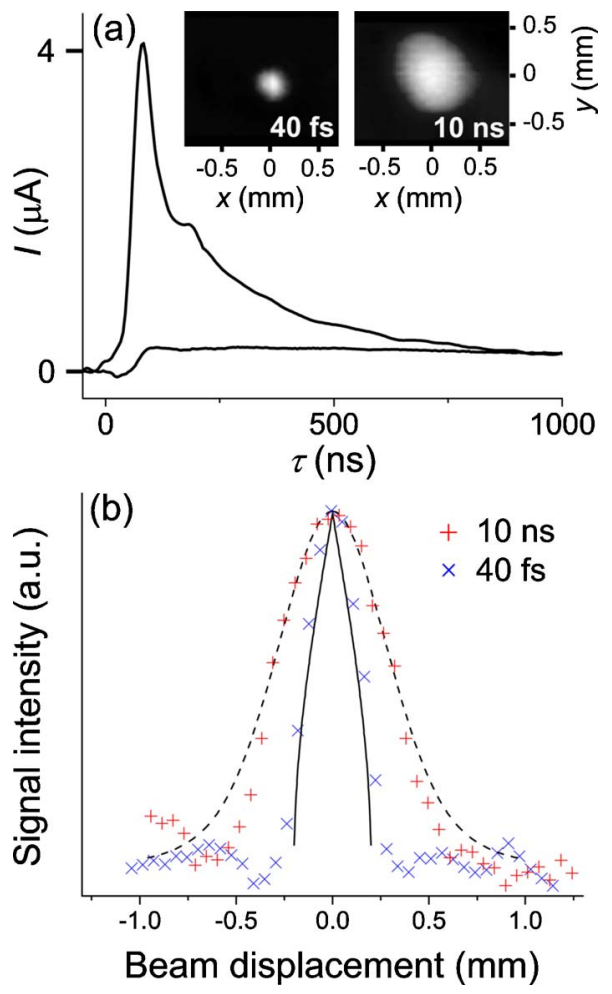

FIG. 2. (Color online) (a) $I(\tau)$ signals measured using the $40 \mathrm{fs}$ and $10 \mathrm{~ns}$ laser excitations (upper and lower lines, respectively). The insets show dependence of the normalized peak current on the excitation beam position. (b) Integrated signal intensity vs beam displacement for femtosecond and nanosecond excitation sources. Solid/dashed lines show the calculated position dependence of coherent/incoherent phonon momentum flux.

$250 \pm 25 \mathrm{~nm}$ substrate. Therefore, in the absence of an applied bias, we determine that the detected current pulse is due to carrier transport induced by the incident phonons. The signal is composed of a fast pulse, about $30 \mathrm{~ns}$ in width, followed by a slowly decaying secondary tail extending over several microseconds.

Ultrafast optical excitation of a metal film is well known to result in a picosecond strain pulse $\mathrm{e}^{1-5}$ and a microsecond heat pulse resulting from the subsequent, relatively slow, thermal relaxation of the metal film. Both contributions consist of acoustic phonons, but the first is a coherent hypersound wavepacket, while the second contains mostly incoherent terahertz phonons. The latter has previously been shown to give rise to "ballistic phonon drag"10 where the acoustic phonons propagating through a semiconductor impart momentum to electrons/holes via carrier-phonon scattering.

To establish the contribution of the picosecond strain pulse to the observed charge transport, we compared the signal due to attenuated 40 fs laser pulses with that induced by $10 \mathrm{~ns}$ pulses from the Nd:YLF source. Laser pulses from each source were of the same energy $\left(8 \mathrm{~mJ} / \mathrm{cm}^{2}\right)$, spot size, and beam profile, but the peak power within the $10 \mathrm{~ns}$ pulse is far below that required to generate a coherent strain pulse, allowing the observation of the electrical signal induced by an incoherent phonon heat pulse alone. Figure 2(a) shows $I(\tau)$ measured using the two different laser sources. The nanosecond source induces a small current (peak $\sim 0.3 \mu \mathrm{A}$ ) in the sample (lower line) whose magnitude and long decay time is qualitatively consistent with the signal observed at later times when using the femtosecond excitation. The greater amplitude of the signal induced by the femtosecond laser (upper line) can be clearly seen. Moreover, the fast rising edge and magnitude of the fs laser signal is certainly limited by the bandwidth of the electronics $(<500 \mathrm{MHz})$ whereas the rising edge of the signal from the nanosecond laser is beneath the electronic bandwidth ceiling. Thus, the difference in the peak induced current is possibly several orders of magnitude larger than is observed. As was demonstrated in Ref. 11, where superconducting bolometers with nanosecond response time were used to detect the phonon flux induced by femtosecond excitation of a metal film, for incident power densities similar to those used here, the initial part of the phonon packet is dominated by the strain pulse which travels at or very slightly faster (depending on strain amplitude) than the speed of low-frequency LA sound. A few nanoseconds later, the phonon packet is dominated by incoherent LA phonons which have a spread of flight times due to scattering and acoustic anisotropy. This raises the possibility that the strong initial peak in the femtosecond data is due to the strain pulse.

To distinguish the contributions from coherent and incoherent phonons, we measured the dependence of the signal on the position of the excitation laser spot with respect to the device. The inset of Fig. 2(a) shows the normalized signal intensity in the initial $50 \mathrm{~ns}$ of the pulse (i.e., we record signal dominated by ballistic phonons) versus laser beam position, plotted as a gray scale image for both laser sources. The $\sim 0.3 \mathrm{~mm}$ spot in the femtosecond laser image follows closely the area of the sample convolved with the laser beam profile, whereas the $\sim 1 \mathrm{~mm}$ diameter spot in the image recorded using the nanosecond laser source is a characteristic of the positional dependence seen in ballistic phonon drag experiments. This is seen more clearly in Fig. 2(b) which shows line scans of the intensity taken through the centre of the images and calculations of the positional dependencies for coherent and incoherent phonons. Owing to its high degree of spatial coherence across the generating area, which is much larger than the typical phonon wavelength, the strain pulse should propagate as a highly collimated beam from the source to the device. The solid line shows the positional dependence calculated as the convolution of the source and device shapes, which follows closely the measured data. The incoherent LA phonons in the heat pulse generated by the nanosecond laser propagate isotropically from each point in the source (phonon focussing effects are very weak for LA phonons in cubic crystals ${ }^{12}$ and so can be ignored here). The dashed line shows the positional distribution of the vertical components of the ballistic phonon momentum flux emanating from a point source, integrated over the laser spot area, and convolved with the device shape. This is also in very good agreement with the measured signal using the ns excitation.

Furthermore, the observed position dependence of the measured signals in Fig. 2 is characteristically similar to previous data using bolometers to detect the coherent hypersound wave packet followed by the incoherent heat pulse. ${ }^{10,13}$ Thus, the data in Fig. 2 lead us to the conclusion that, when using an ultrafast excitation source, the initial part of the current transient is driven mostly by the picosecond strain pulse. Any early arriving components of the incoherent heat pulse would result in a broadening of the spatial dependence and hence must be relatively weak.

to AIP license or copyright; see http://apl.aip.org/apl/copyright.jsp 
Having determined that the initial peak observed in the fs excitation measurement is largely due to the strain pulse, we propose the following explanation of the observed response: due to the coherence of the phonon wavepacket in the picosecond strain pulse, a traveling potential well in the conduction band is produced which propagates through the device at $c_{\mathrm{LA}}$, trapping electrons and dragging them through the crystal. The depth of the well is given approximately by $\varepsilon D$, where $D$ is the deformation potential constant $(\sim 10 \mathrm{eV}$ in GaAs) and $\varepsilon$ is the strain amplitude. Assuming a strain of $10^{-3}$, typical for the laser powers used here, gives a potential well with depth of $\sim 10 \mathrm{meV}$. Without scattering, we estimate the peak current, $I_{p} \approx e n_{W} c_{\mathrm{LA}} A$, where $e$ is the electronic charge, $n_{W}$ is the carrier density trapped in the traveling well (which depends on its depth), and $A$ is the device area. The measured current is given by $I_{p} \tau_{p} / \tau_{e}$, where $\tau_{p} \sim 2$ ps and $\tau_{e} \sim 10 \mathrm{~ns}$ are, respectively, an estimate of the duration of the acoustic pulse and the combined response time of the device and signal acquisition electronics (determined from the measured rise time of the signal). This calculation gives a value of $\sim 10 \mu \mathrm{A}$, which is approximately consistent with the observed value. However, at this stage we cannot exclude effects relating to the interfaces with the heavily $n$-doped contact regions.

We also demonstrate that the coherent strain pulse can also be used to excite a current in a heterostructure. The inset of Fig. 3 shows the current-voltage, $I(V)$, characteristic of the SL device taken at $T=2 \mathrm{~K}$ displaying nonlinearity due to the artificial potential for conduction electrons. Figure 3 shows the induced current transient using a $40 \mathrm{fs} 8 \mathrm{~mJ} / \mathrm{cm}^{2}$ excitation. The features of the signal are qualitatively similar to that recorded using the GaAs epilayer sample with a fast transient beginning at $\tau \sim 55 \mathrm{~ns}$ (consistent with the $275 \pm 25 \mu \mathrm{m}$ substrate thickness), followed by a slowly decaying tail. Scanning the laser across the substrate showed the signal was induced only when the laser was directly opposite the device, suggesting the same mechanism as was observed in the GaAs epilayer sample.

In conclusion, we have demonstrated that it is possible to induce a current in both a $n$-type epilayer and heterostructure semiconductor crystal using an acoustic strain pulse. The strain pulses were generated by ultrafast optical excitation of

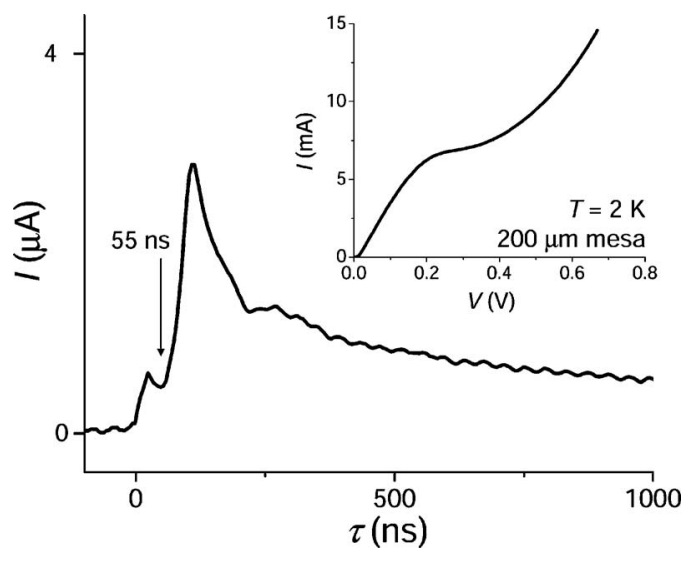

FIG. 3. $I(\tau)$ signal measured in a SL device using the 40 fs excitation with an intensity of $8 \mathrm{~mJ} / \mathrm{cm}^{2}$ at $T=2 \mathrm{~K}$. The $\mathrm{SL} I(V)$ curve is shown in the inset.

an $\mathrm{Al}$ film. This method of driving charge through a semiconductor crystal could be used to study ultrafast carrier dynamics in semiconductor nanostructures as well as having applications in generating picosecond current pulses in semiconductor materials.

The authors acknowledge support for this work from EPSRC of the UK.

${ }^{1}$ C. Thomsen, H. T. Grahn, H. J. Maris, and J. Tauc, Phys. Rev. B 34, 4129 (1986).

${ }^{2}$ G. Tas and H. J. Maris, Phys. Rev. B 49, 15046 (1994).

${ }^{3}$ T. Saito, O. Matsuda, and O. B. Wright, Phys. Rev. B 67, 205421 (2003).

${ }^{4}$ H. Y. Hao and H. J. Maris, Phys. Rev. Lett. 84, 5556 (2000).

${ }^{5}$ O. L. Muskens and J. I. Dijkhuis, Phys. Rev. Lett. 89, 285504 (2001).

${ }^{6}$ R. J. von Gutfeld and A. H. Nethercot, Phys. Rev. Lett. 12, 641 (1964).

${ }^{7}$ A. Huynh, N. D. Lanzillotti-Kimura, B. Jusserand, B. Perrin, A. Fainstein, M. F. Pascual-Winter, E. Peronne, and A. Lemaître, Phys. Rev. Lett. 97, 115502 (2006).

${ }^{8}$ J. F. Robillard, A. Devos, and I. Roch-Jeune, Phys. Rev. B 76, 092301 (2007).

${ }^{9}$ A. V. Akimov, A. V. Scherbakov, D. R. Yakovlev, C. T. Foxon, and M. Bayer, Phys. Rev. Lett. 97, 037401 (2006).

${ }^{10}$ H. Karl, W. Dietsche, A. Fischer, and K. Ploog, Phys. Rev. Lett. 61, 2360 (1988).

${ }^{11}$ N. M. Stanton and A. J. Kent, Phys. Rev. B 73, 220301 (2006).

${ }^{12}$ J. P. Wolfe, Imaging Phonons: Acoustic Wave Propagation in Solids (Cambridge University Press, Cambridge, 1998).

${ }^{13}$ A. J. Kent and N. M. Stanton, J. Phys.: Conf. Ser. 92, 012004 (2007). 